\title{
SU(2) and SU(4) Kondo effect in double quantum dots
}

\author{
Jernej Mravlje*, Anton Ramšak ${ }^{\dagger, *}$ and Tomaž Rejec ${ }^{\dagger, *}$ \\ ${ }^{*}$ Jožef Stefan Institute, Jamova 39, Ljubljana, Slovenia \\ ${ }^{\dagger}$ Faculty of Mathematics and Physics, University of Ljubljana, Jadranska 19, Ljubljana, Slovenia
}

\begin{abstract}
We investigate serial double quantum dot systems with on-site and inter-site interaction by means of Schönhammer-Gunnarsson projection-operator method. The ground state is established by the competition between extended Kondo phases and localized singlet phases in spin and charge degrees of freedom. We present and discuss different phases, as discerned by characteristic correlation functions. We discuss also how different phases would be seen in linear transport measurements.
\end{abstract}

Keywords: Kondo effect, double quantum dots, conductance

PACS: 73.23.-b, 73.63.Kv, 72.15.Qm

\section{INTRODUCTION}

In the last decade the advances in experimental techniques enabled the exploration of intriguing many-body effects occurring in solid-state systems such as the Kondo effect [1] by means of measuring the conductance of nanoscale electrical circuits. Tiny pools of electrons defined by electrodes - quantum dots (QDs) - constitute artificial atoms/molecules. Additional gates enable tuning of the orbital levels as well as the tunneling rates, which makes systematic exploration of various effects experimentally accessible. The Kondo effect is essentially the increased scattering rate (with phase shifts near $\pi / 2$ ) at low temperatures due to magnetic impurities in host metals. In transport experiments through quantum dots it is seen in another disguise: it is discerned as the amplification of the conductance towards unitary limit. Interesting way to proceed further is to analyze the consequences of inter-impurity interaction by looking at the transport through double quantum dot (DQD) systems.

The characteristic feature of the two-impurity Kondo physics is that the two impurities either form an inter-impurity singlet, which is virtually decoupled from conduction electrons or they form a double Kondo state $\mathrm{SU}(2) \times \mathrm{SU}(2)$, in which each spin characterized by the SU(2) symmetry group is screened by the conduction electrons [2] depending on the scales of the energies of the inter-impurity singlet formation $J$ and Kondo state formation $T_{K}$. When the symmetry of the Hamiltonian is larger the Kondo temperature is enhanced. For double quantum dots, which have the capacitative interaction $V$ tuned near the value of the on-dot interaction $U$, the SU(4) Kondo effect occurs [3].

Here we report our results on the competition between extended Kondo and localized singlet phases in serial DQD systems with inter-dot interaction in the point of particlehole symmetry [4] and discuss also the phases which occur outside this point. The SU(4) Kondo phase cannot be explored directly by transport experiment through a DQD as the 
conductance is small irrespective of whether the system is in the SU(4) Kondo state or not. Nevertheless, the scale of the SU(4) condensation energy can be estimated by tuning the system away from the point of SU(4) symmetry until the SU(4) Kondo state collapses. The boundary is easy to discern from the conductance data as the conductance is unity whenever the crossover between the phases takes place.

\section{MODEL AND METHOD}

We model DQDs by the two-impurity Anderson Hamiltonian $H=H_{\mathrm{d}}+H_{1}$, where $H_{\mathrm{d}}$ corresponds to the isolated dots

$$
H_{\mathrm{d}}=\sum_{i=1,2}\left(\varepsilon n_{i}+U n_{i \uparrow} n_{i \downarrow}\right)+V n_{1} n_{2}-t \sum_{\sigma}\left(c_{1 \sigma}^{\dagger} c_{2 \sigma}+\text { h.c. }\right)
$$

with $n_{i}=n_{i \uparrow}+n_{i \downarrow}, n_{i \sigma}=c_{i \sigma}^{\dagger} c_{i \sigma}$. The dots are coupled by a tunneling matrix element $t$ and a capacitive $V$ term. The on-site energies $\varepsilon$ and the Hubbard repulsion $U$ are taken equal for both dots. $H_{1}$ describes the noninteracting left and right tight-binding leads with hopping parameter $t_{0}$ and the coupling of the leads to the DQD. We denote the characteristic tunneling rate of an isolated electron from the dot to the lead by $\Gamma=t^{\prime 2} / t_{0}$, where $t^{\prime}$ is the parameter characterizing the dot-lead hopping.

To calculate the ground state of the system we use the Schönhammer and Gunnarsson projection-operator basis $[5,6]\left|\Psi_{\lambda \lambda^{\prime}}\right\rangle=P_{\lambda 1} P_{\lambda^{\prime} 2}|\tilde{0}\rangle$, which consists of projectors $P_{\lambda i}$; $P_{0 i}=\left(1-n_{i \uparrow}\right)\left(1-n_{i \downarrow}\right), P_{1 i}=\sum_{\sigma} n_{i \sigma}\left(1-n_{i \bar{\sigma}}\right), P_{2 i}=n_{i \uparrow} n_{i \downarrow}$ and additional operators involving the operators in leads. We used up to $\sim 100$ additional combinations of operators consisting of, for example, $P_{3 i}=P_{0 i} \widehat{v} P_{1 i}$, where $\widehat{v}$ denotes the tunneling to/from dot $i$. These operators are applied to the state $|\tilde{0}\rangle$, which is the ground state of the auxiliary noninteracting DQD Hamiltonian of the same form as $H$, but with $U, V=0$, renormalized parameters $\varepsilon, t, t^{\prime} \rightarrow \tilde{\varepsilon}, \tilde{t}, \tilde{t}^{\prime}$ and additional parameter $\tilde{t}^{\prime \prime}$ which corresponds to hopping from left dot to right lead and vice versa which although absent in the original Hamiltonian is present in the effective Hamiltonian in some parameter regimes.

The conductance is calculated using the sine formula [7], $G=G_{0} \sin ^{2}\left[\left(E_{+}-\right.\right.$ $\left.\left.E_{-}\right) / 4 t_{0} L\right]$, where $G_{0}=2 e^{2} / h$ and $E_{ \pm}$are the ground state energies of a large auxiliary ring consisting of $L$ non-interacting sites and an embedded DQD, with periodic and anti-periodic boundary conditions, respectively.

\section{GROUND STATE AND CONDUCTANCE OF DQD WITH INTER-DOT INTERACTION}

\section{Detached DQDs}

The starting point towards the understanding of the ground state of DQDs are the filling properties of isolated DQDs (i.e. of the Heitler-London or the two-site Hubbard model). The first electron is added when $\varepsilon=t$, and the second when $\varepsilon=-t+J+[(U+$ $V)-|U-V|] / 2$, where $J=\left[-|U-V|+\sqrt{(U-V)^{2}+16 t^{2}}\right] / 2$ is the difference between 
singlet and triplet energies. When $n=2$ the ground state is $[\alpha(|\uparrow \downarrow\rangle-|\downarrow \uparrow\rangle)+\beta(|20\rangle-$ $|02\rangle)] / \sqrt{2}$, where $\alpha / \beta=4 t /\left(V-U+\sqrt{(U-V)^{2}+16 t^{2}}\right)$. The range of $\varepsilon$ where single occupation is favorable is progressively diminished when $V \neq U$. For large $t$ or at (and near) $V=U$ the molecular bonding and anti-bonding orbitals are formed as is seen here from $\alpha \sim \beta$.

\section{Attached DQDs and conductance}

As we attach DQDs to the leads the ground state either is or is not reminiscent of the ground state of the isolated system. Here the latter possibility is always due to some kind of the Kondo effect. In the top panels of Fig. 1 the ground state of DQDs are presented with pictograms for $V=0, U$ on the left and right, respectively. The near vertical dividing lines correspond to values of parameters where the ground state of the isolated system is degenerate due to matching energies of states with different occupancies, for example, the rightmost line corresponds to $E(0)=0=E(1)=\varepsilon-t$. The horizontal U-shaped line is given by $J=2.2 T_{K}$, where the scale of the Kondo condensation energy is estimated by $T_{K}=\sqrt{U \Gamma / 2} \exp (-\pi \varepsilon(\varepsilon+U) / 2 \Gamma)$ for $U / \Gamma=15$.
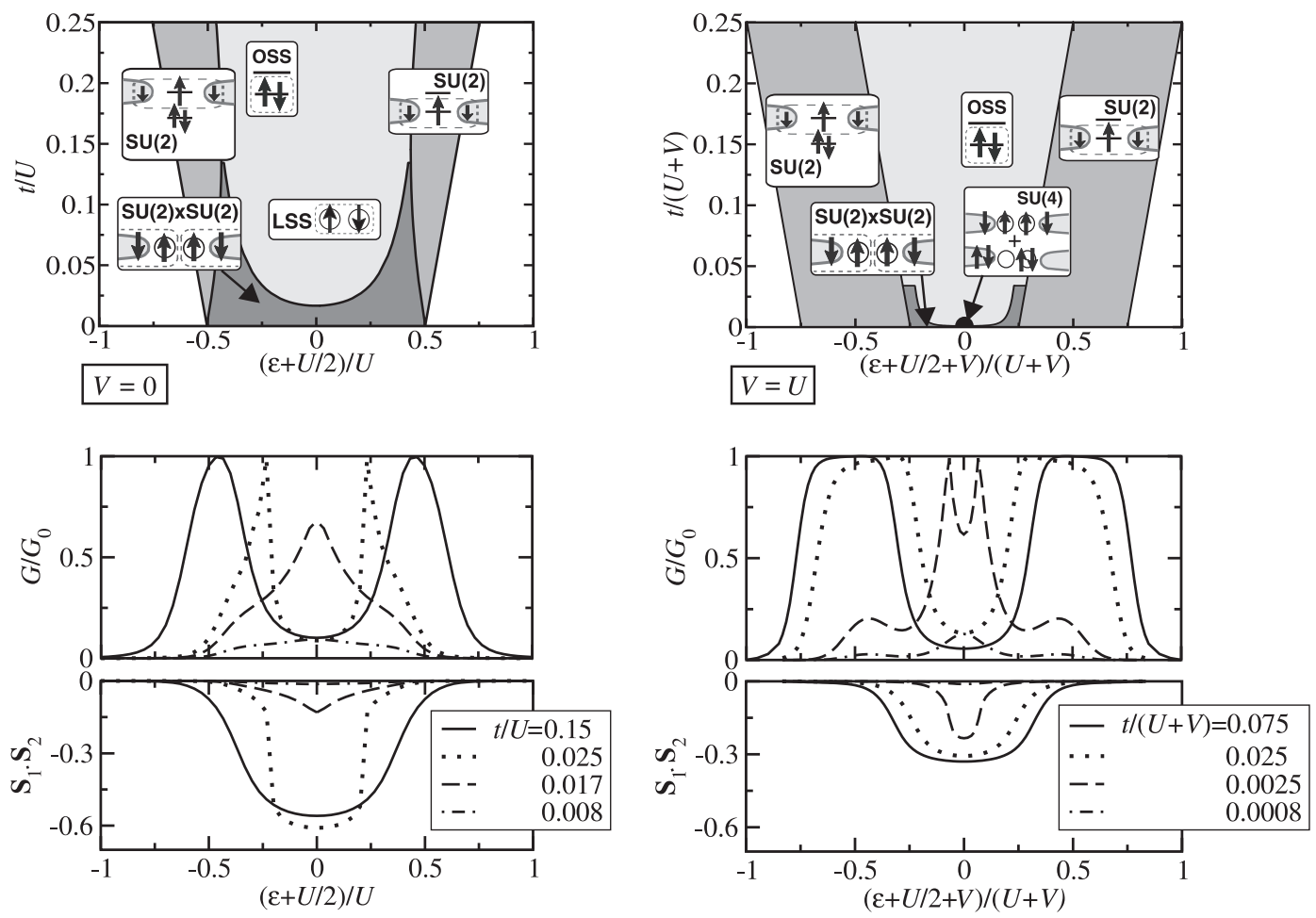

FIGURE 1. - Top panels: phases of serial DQDs for $V=0$ (left) and $V=U$ (right). The occupancy of the DQD falls from left to right. Extended Kondo phases (with leads in pictograms) and localized singlet phases (without leads in pictograms) occur. - Bottom panels: Conductance and spin-spin correlation of the DQD for $t$ above (full and dotted lines for $V=0$; full, dotted and dashed lines for $V=U$ ) and below the localized singlet formation threshold. Note the approaching of $\mathbf{S}_{1} \cdot \mathbf{S}_{2}$ towards $-3 / 8$ for large $t$ indicating the formation of the orbital singlet. 
For $n=0,4$ interaction between electrons (or holes) is not important, hence the ground state is not interesting. For $n=1,3$ the ground-state of the isolated DQD is a free spin in (anti-)bonding orbital, which is, when the leads are attached, at low-temperatures screened by conduction electrons as in 'ordinary' single impurity Anderson model. The most interesting part of the diagrams corresponds to $n \sim 2$. Here the ground state of the isolated system is a non-degenerate singlet but the tunneling to the leads breaks this singlet whenever roughly twice the Kondo condensation energy exceeds the triplet excitation energy $J$. For $V \sim U$ the $J$ is enhanced hence the area corresponding to $\mathrm{SU}(2) \times \mathrm{SU}(2)$ Kondo is diminished. Near the symmetric point, however, another kind of the Kondo effect arises for $V \sim U$ as a consequence of larger symmetry of the $V=U$ Hamiltonian, which partially restores the occurrence of the Kondo phase.

\section{Symmetries}

The Kondo effect occurs as the consequence of the degeneracy of states of isolated impurities. If one looks at the ground state of two isolated impurities coupled by a capacitative (but not tunneling) term $V=U$, one sees that the 6 states $\left|\sigma_{1} \sigma_{2}\right\rangle$, $|20\rangle$ and $|02\rangle$ are degenerate. Indeed, by introducing the pseudospin operator [8] $\tilde{T}^{i}=$ $1 / 2 \sum_{l l^{\prime}=1,2} \sum_{\sigma} c_{l \sigma}^{\dagger} \tau_{l l^{\prime}}^{i} c_{l^{\prime} \sigma}$, where $\tau^{i}$ are the Pauli matrices, and the combined spinpseudospin operators $W^{i j}=S^{i} \tilde{T}^{j}$, one sees that the Hamiltonian is SU(4) symmetric. As long as the $\mathrm{SU}(4)$ symmetry breaking terms are small enough $V-U, t \lesssim T_{K}[S U(4)]$, the ground state is an SU(4) 'spin' screened by the electrons in the leads.

\section{Orbital representation}

A complementary way is to rewrite the Hamiltonian in the basis of orbital operators $c_{b, a}=\left(c_{1} \pm c_{2}\right) / \sqrt{2}$

$$
H_{\mathrm{d}}=\sum_{\alpha=a, b}\left[\varepsilon_{\alpha} n_{\alpha}+\frac{U+V}{2}\left(n_{\alpha \uparrow} n_{\alpha \downarrow}+n_{\alpha \uparrow} n_{\bar{\alpha} \downarrow}\right)\right]+V \sum_{\sigma} n_{a \sigma} n_{b \sigma}+\frac{U-V}{2}\left(C_{\text {flip }}-S_{\text {flip }}\right)
$$

where notation $\bar{a}=b, \bar{b}=a$ is used. The last term of $H_{\mathrm{d}}$ consists of isospin-flip $C_{\text {flip }}=$ $T_{a}^{+} T_{b}^{-}+$h.c. and spin-flip $S_{\text {flip }}=S_{a}^{+} S_{b}^{-}+$h.c. operators, where $S_{\lambda}^{-}=c_{\lambda \downarrow}^{\dagger} c_{\lambda \uparrow}=\left(S_{\lambda}^{+}\right)^{\dagger}$ are spin and $T_{\lambda}^{-}=c_{\lambda \uparrow} c_{\lambda \downarrow}=\left(T_{\lambda}^{+}\right)^{\dagger}$ isospin lowering and raising operators for the orbitals $\lambda=b, a$ (or sites $\lambda=1,2$ ). The full spin (isospin) algebra is closed with operators $S_{\lambda}^{z}=\left(n_{\lambda \uparrow}-n_{\lambda \downarrow}\right) / 2$ and $T_{\lambda}^{z}=\left(n_{\lambda}-1\right) / 2$, respectively.

When $V=U$, the spin- and isospin-flip terms in $H_{\mathrm{d}}$ are absent: the Hamiltonian is mapped exactly to the two-level Hamiltonian with intra- and inter-level interaction $U$ with the bonding and anti-bonding levels coupled to even and odd transmission channels, respectively. When $V \neq U$ this mapping is no longer strictly valid: the electrons try to avoid the inter-level repulsion by occupying aligned spin-states in different orbitals, and the isospin-flip terms induce the fluctuations of charge between orbitals. Both mechanisms prohibit electrons from occupying the well-defined orbital states. 


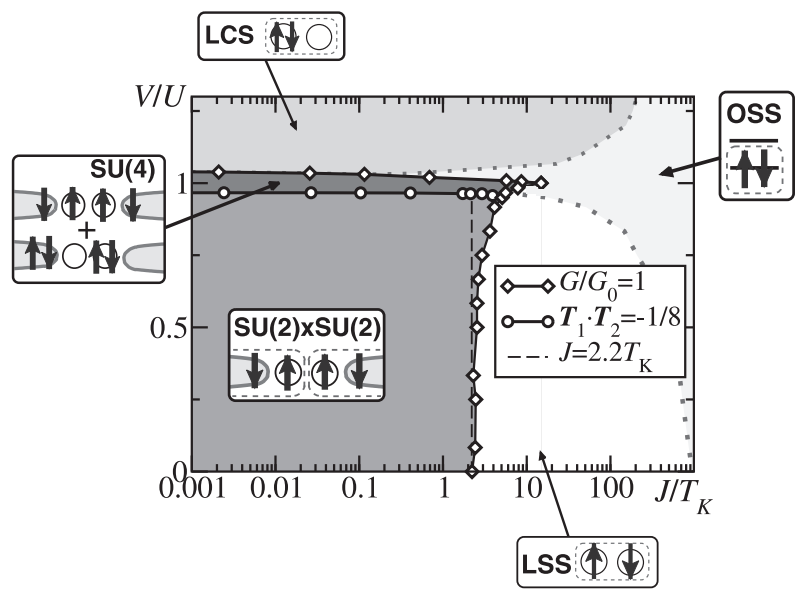

FIGURE 2. Phases of DQD in the point of particle-hole symmetry. The boundaries between Kondo and localized singlet phases are given by peaks in conductance and abrupt changes in correlation functions. The boundaries of the orbital spin singlet state are given by $\mathbf{S}_{1} \cdot \mathbf{S}_{2}=-3 / 16$ and $\Delta n_{1}^{2}=\Delta n_{b}^{2}$ on the upper and lower side, respectively. Note the extension of the Kondo phase behind the line $J=2.2 T_{K}($ dashed) at $V \sim U$.

\section{Numerical results}

In the lower panels of Fig. 1 the conductance and inter-dot spin-spin correlations are plotted. Note that the orbital picture is indeed more robust for the $V=U$ case as indicated by the broad plateaus in conductance corresponding to the SU(2) Kondo effect of a spin residing in the (anti-)bonding orbitals. Moreover, $J$ is enhanced when compared to the $V=0$ case: absence of singlet phase signalled by no peak with unitary conductance and minor spin-spin correlation for all $\varepsilon$ occurs only for smaller $t$. Note also that conductance is small whenever the ground state is practically geometrically separable into parts. In that case the flux can be transported out of the auxiliary ring through the boundary between the parts, yielding zero conductance in our approach [7]. In Fig. 2] we indicate the phases in the $\left(\mathrm{J} / T_{K}, V / U\right)$ plane. Details are given in Ref. [4].

\section{ACKNOWLEDGMENTS}

We acknowledge the support of SRA under grant Pl-0044.

\section{REFERENCES}

1. A. C. Hewson, The Kondo Problem to Heavy Fermions, Cambridge University Press, 1993.

2. B. A. Jones, C. M. Varma, and J. W. Wilkins, Phys. Rev. Lett. 61, 125-128 (1988).

3. M. R. Galpin, D. E. Logan, and H. R. Krishnamurthy, Phys. Rev. Lett. 94, 186406 (2005).

4. J. Mravlje, A. Ramšak, and T. Rejec, Phys. Rev. B 73, 241305(R) (2006).

5. K. Schönhammer, Phys. Rev. B 13, 4336 (1976).

6. O. Gunnarsson, and K. Schönhammer, Phys. Rev. B 31, 4815 (1985).

7. T. Rejec, and A. Ramšak, Phys. Rev. B 68, 035342 (2003).

8. L. D. Leo, and M. Fabrizio, Phys. Rev. B 69, 245114 (2004). 